\title{
Chaine de valeur riz en Afrique de l'Ouest : Performance, enjeux et défis en Côte d'Ivoire, Guinée, Libéria, Mali, Sierra Léone et Sénégal
}

\section{REVIEW PAPER}

\author{
Amadou Abdoulaye FALL, Ph.D. \\ Chercheur à l'Institut Sénégalais de Recherches Agricoles (ISRA) CRA de Saint-Louis, Jardin d'Essai Sor Saint- \\ Louis, Sénégal \\ Correspondance email: aafall22@yahoo.fr
}

Original submitted in on $21^{\text {st }}$ September 2018. Published online at www.m.elewa.org on $31^{\text {st }}$ October 2018 https://dx.doi.org/10.4314/jab.v130i1.5

\section{RÉSUMÉ}

L'objectif de l'étude est d'analyser la structuration de la chaine de valeur riz en Afrique de l'Ouest en vue d'identifier les facteurs de performance, de relever les enjeux et défis. L'étude consiste en une évaluation du potentiel des activités de production, de transformation et de commercialisation liées aux besoins du marché. Le cadre d'analyse est " chaîne des valeurs » basée sur une approche institutionnelle de gouvernance, de structuration, de conduite et de performance. La Structure réfère à la cartographie des acteurs. La Conduite met en œuvre le fonctionnement du système, le rôle des différents acteurs et les fonctions de soutien. La Performance au fonctionnement du marché procède à une analyse coût / bénéfice pour chaque groupe d'acteurs. L'étude indique que la riziculture repose essentiellement sur de petites exploitations familiales avec une superficie moyenne variant entre 0,25 et 4 ha selon les écologies et les pays. Ce diagnostic révèle une émergence des flux de commerce du riz local avec un intéressement de la demande locale. II n'en demeure pas moins que l'offre locale demeure insuffisante pour la majorité des pays. Les services de soutien de la chaine de valeur sont souvent inopérants. La recherche est peu dotée en ressources humaines et financières et ses infrastructures sont vétustes. L'outil de conseil est inapproprié pour une grande cible de producteurs analphabètes. Le secteur de financement est peu impliqué et souvent inopérant par rapport aux produits offerts vis-à-vis de la demande. La chaîne de valeur riz est compétitive, mais durablement peu assurée. La capacité locale à approvisionner le marché ne progresse pas aussi rapidement que la demande. Cela s'explique par de diverses difficultés dans la production avec des rendements très faibles, des frais de transaction élevés et d'une consommation intérieure en croissance permanente. Les défis majeurs restent l'accroissement de l'offre locale et sa qualité par rapport à la demande du marché, l'amélioration de l'usinage et la mise en marché dans des conditions de compétitivité durable.

Mots clef : chaine de valeur, riz, acteurs, coût, marge, offre, demande, échange, performance 
Rice value chain in West Africa: Performance, issues and challenges in Côte d'Ivoire, Guinea, Liberia, Mali, Sierra Leone and Senegal

\begin{abstract}
The objective of the study is to analyze the structure of the rice value chain in West Africa in order to identify the factors of performance, to address the issues and challenges. The study consists of an assessment of the potential of the production, processing and marketing activities related to market demand. The framework of analysis is "value chain" based on an institutional approach to governance, structure, conduct and performance. The Structure refers to the mapping of actors. Conduct implements the functioning of the system, the role of different actors and the support functions. The Performance of the market performs a cost-benefit analysis for each group of actors. The study indicates that rice farming is mainly based on small family farms with an average area of 0.25 and 4 ha depending on ecologies and countries. This diagnosis reveals the emergence of local rice trade flows with an interest in local demand. The fact remains that the local supply is still insufficient for the majority of countries. The support services of the value chain are often inoperable. Research is poorly endowed with human and financial resources and its infrastructure is dilapidated. The extension tool is inappropriate for a large target of illiterate producers. The financing sector is little involved and often inoperant compared to the products offered with regard to demand. The rice value chain in West Africa is competitive, but uninsured. The local capacity to supply the market does not progress as quickly as demand. This is due to various difficulties in production with very low yields, high transaction costs and domestic consumption in constant growth. The major challenges remain the increase of local supply and its quality in relation to market demand, the improvement of milling and marketing in conditions of sustainable competitiveness.
\end{abstract}

Keywords: value chain, rice, actors, cost, margin, supply, demand, exchange, performance

\section{CONTEXTE ET JUSTIFICATIFS}

Le riz joue un rôle important dans l'économie des pays ouest africains et dans la consommation des ménages urbains et ruraux. En outre, du fait de la facilité et des faibles coûts de sa préparation, des prix bas et de la régularité de son approvisionnement, le riz est devenu une denrée de consommation courante et un produit stratégique pour une bonne partie de la population mondiale (FALL, 2007; AfricaRice, 2012). Dans cette perspective, le riz fait partie des cultures ciblées vu son rôle de premier plan dans les stratégies de consommation alimentaire en Afrique de l'Ouest et notamment en Côte d'ivoire, Guinée, Libéria, Mali et Sénégal. De ce point de vue, sa disponibilité permanente doit être assurée pour faire face aux impératifs de sécurité alimentaire. Cependant, la crise alimentaire mondiale de 2008 a montré les limites de l'offre locale et la vulnérabilité du système. L'accroissement des prix des céréales induit de cette crise, a fortement impacté les populations dépendantes en majorité des importations. Ainsi des initiatives ont été prises par les décideurs politiques et leurs partenaires au développement sur l'intensification de la production du riz en vue d'apporter " une réponse à la crise alimentaire mondiale " durant ces dix dernières années. Cependant, la cohérence des stratégies d'intensification de la production ne saurait prospérer sans une connaissance approfondie sur les marges de progrès des divers acteurs en relation avec la demande du marché. Dans le contexte actuel très concurrentiel, la performance de nombreuses entreprises de commerce, de transformation, de services, etc. qui se sont implantées dans les zones de production reste très dépendante du niveau et de la qualité de l'offre de production, mais aussi des stratégies de collecte, de transformation et de mise en marché. La rentabilité financière de ces entreprises est dépendante les unes des autres. L'objectif de cette étude est d'identifier les facteurs déterminants de performance, les potentialités et stratégies de mise en œuvre sur les facteurs aussi bien stimulants que sur les goulots d'étranglement des divers maillons de la chaine au niveau des pays ciblés. 

défis en Côte d'Ivoire, Guinée, Libéria, Mali, Sierra Léone et Sénégal

\section{METHODOLOGIE}

Échantillonnage : Dans la chaîne de valeur, il est noté des acteurs (producteurs, transformateurs et commerçants) et d'acteurs indirects ou services d'approvisionnement. Vues l'étendue et l'hétérogénéité des zones de production, de transformation et de commercialisation du riz local dans les pays cibles, la méthode d'échantillonnage dite « multi-stage » a été utilisé auprès des acteurs directs. Dans tous les pays, trois des sites les plus représentatifs du système rizicole ont été identifiés avec les experts du pays selon notamment les critères de (i) de zones à forte potentialités de production et (ii) d'opportunités d'accès au marché et de transformation. Ensuite, le choix aléatoire est procédé sur les producteurs, commerçants et transformateurs dans les sites retenus. L'échantillon global sur les acteurs directs est de 2450 acteurs dans les six pays ciblés. La taille de l'échantillon des producteurs varie entre 240 et 600 par pays, celle des transformateurs à 30 par pays et celle des commerçants est de 50 par pays. Pour le choix des unités d'observation sur les acteurs indirects, il a été procédé par un choix raisonné sur 1 à 10 personnes ressources avec notamment un guide d'entretien et des focus groupe dans les sites visités par pays. Ces enquêtes ont été complétées par la collecte des données agrégées (prix, productions, importations, etc.) auprès des structures statistiques et de marchés et la documentation.

Cadre analytique Chaine des valeurs : La Chaine de Valeurs s'appuie sur les différentes composantes de la filière en y ajoutant les concepts de gouvernance, de structuration, de conduite et de performance. L'étude consiste en une évaluation du potentiel des activités de production, de transformation et de commercialisation liées aux besoins du marché en vue d'évaluer leur marge de progrès et leurs contraintes selon la Structure, la Conduite et la Performance. La Structure réfère à l'identification et la typologie des acteurs à chaque maillon. La Conduite met en œuvre le fonctionnement du système (type de marché, prix, préférences des consommateurs, offre et demande) et le rôle des différents acteurs et les fonctions de soutien et de service. Les deux premières composantes mènent vers des choix des chaines et de Gouvernance. Ensuite viennent la Performance et le renforcement de capacité avec la distribution de la Valeur Ajoutée (figure 1). Cette composante procède à l'analyse coût/bénéfice pour chaque groupe d'acteurs et met en jeu le fonctionnement du marché sur le triptyque : efficacité (accès aux intrants, au crédit, aux ressources, à la collaboration.), efficience de la filière (coûts de transaction, distribution du profit, disponibilité du produit) et les contraintes et opportunités.

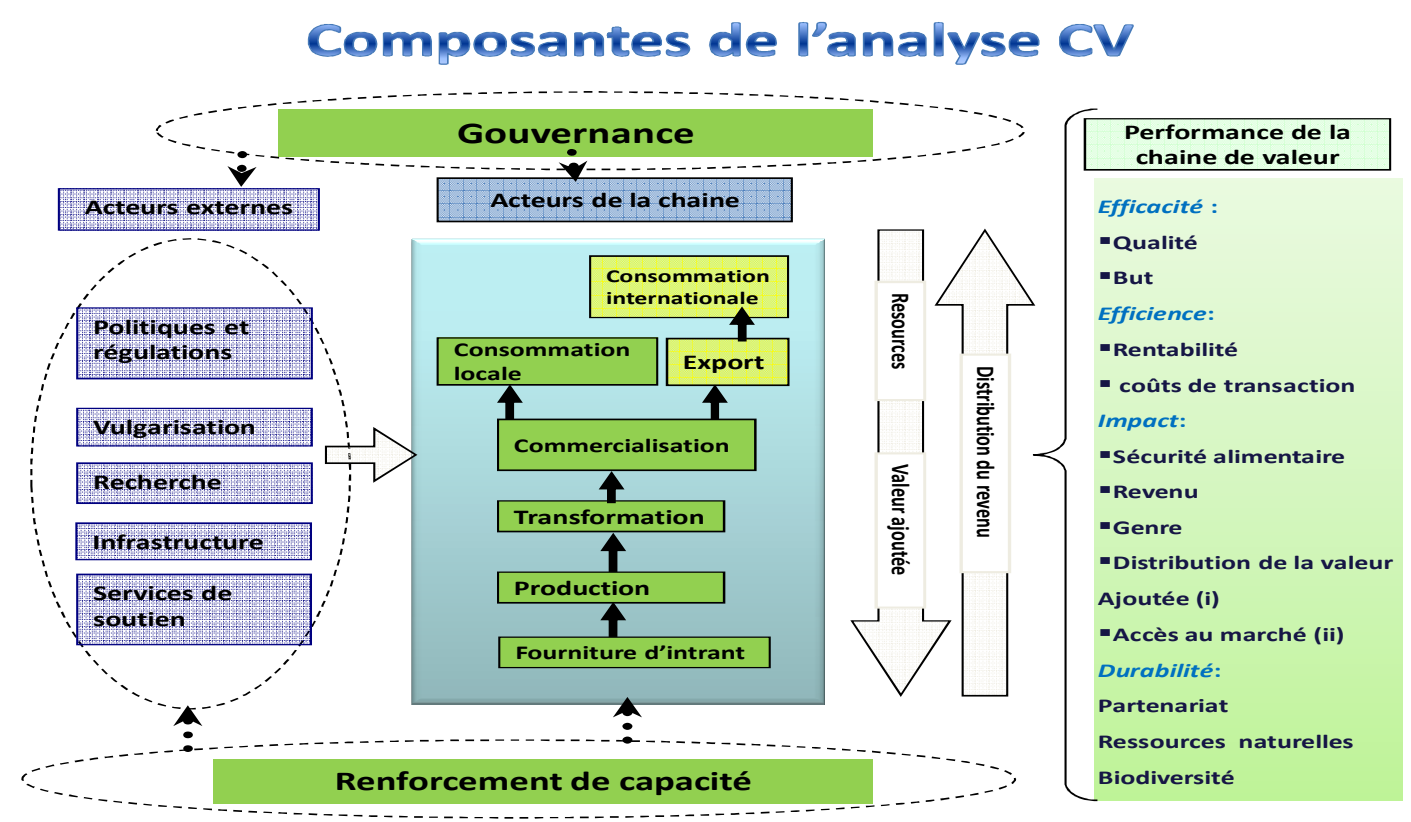

Figure 1 : Schéma de la chaine de valeur

Source : Auteur. 


\section{SYSTEMES DE PRODUCTION ET ENVIRONNEMENT INSTITUTIONNEL}

En Afrique de l'Ouest, de grands bassins de production de riz sont au long du golfe de guinée, au Mali, au Sénégal., avec des emblavures, des niveaux de productions et de rendements très différents d'une zone à une autre. Les systèmes de production rizicole sont cependant, nombreux et diversifiés. Dans le long du golfe de Guinée, le riz de mangrove et de bas-fonds prédomine dans les zones côtières. Cependant, dans les zones de plateaux comme en Guinée, dans le Sud du Mali et au Sud du Sénégal le riz pluvial est dominant. Le riz irrigue occupe trois grandes zones : la vallée du fleuve Sénégal, l'Office du Niger au Mali, et l'État de Kano au Nigeria. Dans les vallées de ces pays, des périmètres rizicoles de petite taille se développent le long des cours d'eau avec l'aménagement ou non des bas-fonds. Les bas-fonds s'érigent ainsi comme des fonds des vallons et des petites vallées. Ils constituent les grands axes de drainage des eaux y comprise les eaux de ruissellement avec une concentration des nappes phréatiques.

La riziculture pluviale stricte couvre plus de 2 millions d'hectares dans les savanes humides où l'alimentation en eau des plantes. Elle est assurée uniquement par les eaux des pluies avec une productivité faible (rendement moyen de 1,3 t/ha). Dans ce système, le travail est encore manuel sur de petites surfaces, sans engrais ni matériels végétales améliorés. Toutes les opérations de récolte et post récoltes sont manuelles. Dans notre zone d'intervention, elle varie par pays et par saison. Elle représente $92 \%$ des superficies cultivées et compte pour $73 \%$ de la production en Côte d'Ivoire avec une dominance à l'Ouest, au Nord et au Centre-Ouest du pays. En Guinée, elle représente $65 \%$ des superficies emblavées et contribue à $64 \%$ de la production. Le système est dominant en Guinée forestière avec plus de 89\% des emblavures (IRAG, 2016). Au Sénégal, il est caractérisé par un système extensif et couvre les régions du centre, du sud et du sud-est et représentent $23 \%$ des emblavures en 2017. En Sierra Leone, le riz est cultivé par plus de $96 \%$ des petites exploitations agricoles familiales (figure 2) à presque totalement sur le pluvial avec deux écologies le plateau et les bas-fonds. Au Mali, le pluvial strict est pratiqué dans les régions sud avec seulement $3 \%$ des emblavures de riz. Au Libéria, par contre le riz est une culture de subsistance particulièrement cultivé sur brulis dans les systèmes de plateau représentant 95\% (figure 2).

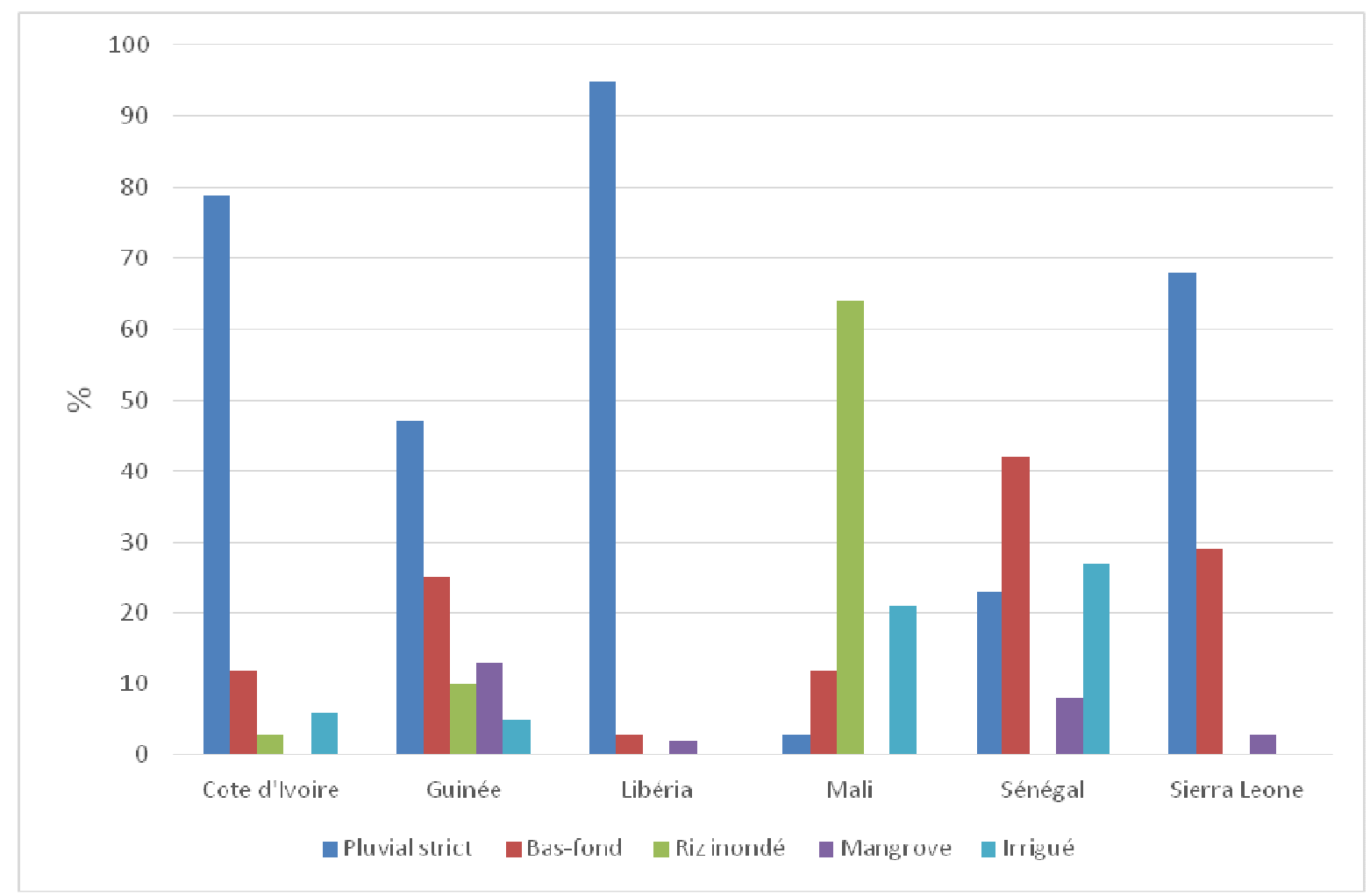

Figure 2 : Répartition des superficies en riz (\%) par écologie en Afrique de l'Ouest Source : Enquêtes CORAF/WECARD, 2014 (Données compilées par pays en 2017). 

défis en Côte d'Ivoire, Guinée, Libéria, Mali, Sierra Léone et Sénégal

La riziculture de bas-fonds essentiellement dans les zones humides couvrant aussi plus de 2 millions d'hectares avec un rendement moyen de 1,85 t/ha. lls se définissent comme des fonds des vallons et des petites vallées. En Guinée, elle constitue $25 \%$ des emblavures. Ce système de production produit en moyenne entre 1,5 et 2,5 t/ha contribuant à hauteur de $9 \%$ de la production rizicole du pays. Au Mali, elle est caractérisée par une grande diversité et représente $12 \%$ des emblavures. Elle est pratiquée en général par les femmes dans les régions de Ségou, Sikasso et Kayes avec en moyenne 0,8 à 2 t/ha. En Sierra Leone, $29 \%$ des emblavures sont dans les diverses écologies de basfonds. Les pratiques culturales sont très rudimentaires pour la majorité des agriculteurs qui sont sous-équipés et ont un accès difficile aux intrants. Le système est très faiblement cultivé au Libéria (3\%) et le riz est principalement destiné à l'autoconsommation à l'échelle du ménage ou du village. En Côte d'Ivoire, les bas-fonds sont aménagés et représentent $5 \%$ des superficies. Ce système se localise au Centre, à l'Ouest, au CentreOuest et au Nord du pays. Au Sénégal, elle représente $42 \%$ des emblavures, largement le plus dominant des systèmes rizicoles, mais destinée principalement à l'autoconsommation. Elle est pratiquée principalement dans la Casamance naturelle (régions de Ziguinchor et de Sédhiou) et exclusivement par les femmes.

La riziculture irriguée est avec maîtrise totale de l'eau couvrant une superficie 516000 ha, soit moins de $9 \%$ des terres emblavées en Afrique de l'Ouest dont les rendements moyens sont estimés à 3,6 t/ha avec des pics de $8 \mathrm{t} / \mathrm{ha}$. Au Mali, les superficies en riz cultivées en submersion contrôlée sont de l'ordre de 45000 ha essentiellement dans les régions de Ségou et de Mopti. En maîtrise totale de l'eau, la région notamment de Ségou avec l'Office du Niger couvre plus de 100000 ha avec des rendements moyens de 5 à 6 t/ha. En Guinée, ce sont des basfonds aménagés ainsi tributaire des pluies pour l'installation du riz et représente $5 \%$ des emblavures. Elle contribue $7,2 \%$ à la production nationale. En Côte d'Ivoire, elle représente $6 \%$ des emblavures. Le riz irrigué est cultivé dans les bas-fonds aménagés ou les barrages au Centre, à l'Ouest, au
Centre-Ouest et au Nord du pays. Ce système à forte mécanisation et de consommation d'intrants produit en moyenne $3,5 \mathrm{t} / \mathrm{ha}$ et contribue ainsi à hauteur de $21 \%$ de la production nationale. En Sierra Leone, c'est plutôt le riz inondé dans les vallées des bas-fonds qui est pratiqué, de même qu'au Libéria. Dans ces pays en pleine restructuration de leur secteur agricole, des investissements en ouvrages hydro-agricoles ne sont pas encore réalisés. Au Sénégal, elle occupe $27 \%$ des superficies rizicoles et contribue entre 50 et $65 \%$ de la production nationale dont $69 \%$ sont destinés à la commercialisation. Le système est fortement mécanisé et intensif produisant des rendements moyens de 6,5 t/ha avec des pics de 8-9 tha.

La riziculture de mangrove correspond à un lieu de rencontre de deux types d'eau : l'eau douce et l'eau salée. Elle est estimée à $4 \%$ des superficies emblavées en riz en Afrique de l'Ouest soit 240000 ha avec un rendement moyen actuel de 2t/ha (Boutsen et Aertsen, J., 2013). En Guinée, elle se trouve en Guinée Maritime et représente 13\% des superficies emblavées en 2017 et produit des niveaux de rendement les plus élevés entre 1,5 et $3,5 t / h a$. Sa part dans la production nationale du riz est de 8\%. En Sierra Leone, elle occupe 3\% des superficies en riz et contribue pour $10 \%$ de l'offre locale, tandis qu'elle représente $2 \%$ au Libéria. Le système est cependant absent au Mali et en Côte d'Ivoire qui privilégie respectivement le système irrigué et le riz de plaine inondée. Le Sénégal enregistre $8 \%$ de ces terres emblavées en riz en la mangrove dans les régions de Casamance ; Fatick et Kaolack. En Afrique de l'Ouest le nombre d'hectares emblavés en riz est passé de 3 millions dans les années 1980 à plus de 8 millions en 2017 (FAO, 2017). La Guinée est le deuxième derrière le Nigéria avec 1685056 d'hectares emblavés en riz en 2017. Viennent ensuite la Sierra Leone avec 754 113 ha, la Cote d'Ivoire avec 703413 ha durant la même période. Dans l'optique de réduire la dépendance des pays de l'Afrique de l'Ouest vis-àvis des importations, le riz fait l'objet depuis des décennies d'une préoccupation constante des autorités politiques. Ainsi, au cours de ces dix (10) dernières années, l'environnement institutionnel du 

défis en Côte d'Ivoire, Guinée, Libéria, Mali, Sierra Léone et Sénégal

secteur rizicole en Afrique de l'Ouest a été marqué par un ensemble de politiques de promotion et de programmes de soutien à la production. En réaction à la crise de 2008, les états de l'Afrique de l'Ouest et leurs partenaires ont lancé des programmes de soutien à moyen terme à la production. Naissent, entre autres, I'Initiative Riz au Mali, la Grande Offensive Agricole pour la Nourriture et l'Abondance au Sénégal, les programmes d'autosuffisance en riz en Guinée, en Côte d'Ivoire, Sierra Leone et Sénégal. Les bailleurs de fonds, pour leur part, ont

\section{PERFORMANCE DE LA CHAINE DE VALEUR DU RIZ} Situation de l'offre et de la demande: L'Afrique de l'Ouest, avec une production moyenne (2013-2017) de 14700504 millions de tonnes de riz paddy, importe en moyenne durant la même période environ 7210754 millions de tonnes de riz blanchi pour couvrir des besoins de consommation (USDA, 2018). Elle est de loin la première en Afrique en termes de production et de consommation durant ces cinq dernières années. Sa production est passée de 13,5 millions de tonnes en 2013 à 16,6 million en 2017 soit $53 \%$ de la production du continent (idem, 2018). Cette production est essentiellement concentrée dans les bassins du Nigéria $(40 \%)$, de la Guinée et du Mali (30\%), de la Côte d'Ivoire et du Libéria (10-15\%). La part de la consommation de riz par rapport à l'ensemble des céréales est d'environ $26 \%$. Cette demande ne cesse d'augmenter avec l'accroissement de sa population en moyenne de $3 \%$ par an. La croissance annuelle de la production de riz est par contre de l'ordre de $22 \%$ avec une consommation per capita qui croît de 5 à $6 \%$ par an sur la période (USDA, 2018). Malgré l'accroissement des productions du riz en Afrique de l'Ouest, l'offre ne satisfait pas la demande intérieure. Une analyse des tendances de la production, du rendement et des superficies du riz de 2013 à 2017 montre une évolution en dents de scie et en trajectoires différentes selon les pays.

La production moyenne de la Sierra Léone, entre 2013 et 2017 est de 1206610 millions de tonnes paddy pour une superficie moyenne récoltée de 639579 ha, tandis que la Côte d'ivoire a produit 1871782 millions de tonnes sur 763908 ha emblavés durant la même période. En Guinée, la production a atteint les 1979369 millions tonnes avec seulement une superficie moyenne emblavée de 1611425 ha entre 2013 et 2017. Durant la même période au Libéria, la superficie est en moyenne de 214082 ha pour une production de 278628 tonnes. $\mathrm{Au}$ Mali et au Sénégal, les superficies moyennes adopté des programmes, tels que le « Food Facility " de I'Union européenne, la coalition de développement du riz en Afrique (CARD) avec le Japon, le «Feed the Future » de I'USAID, le Programme d'urgence du riz pour I'Union Économique et Monétaire Ouest Africaine (UEMOA), etc. Ceci témoigne du renouveau d'intérêt des États et des bailleurs de fonds pour les questions d'agriculture et de sécurité alimentaire » (Del Villar et al., 2013).

emblavées en riz de 2013 à 2017 sont estimées respectivement à 834643 ha et 211089 ha pour des productions d'environ 2780905 et 751189 de tonnes paddy. Cependant, l'offre locale de la plupart des pays à l'exception du Mali reste fragile. Malgré cette hausse des productions entre 2013 et 2017 induites par l'accroissement des superficies emblavées en riz, la production nationale de la plupart des pays ciblés se situe entre 20 et $60 \%$ de la demande intérieure. Les importations couvrent le reste des besoins de la consommation. Dans les importations en Afrique de l'Ouest, ce sont le Nigéria (1,9 million t/an), la Cote d'Ivoire (1,35 million t.an) et Sénégal (954 000 t/an) qui dominent. Le Mali constitue le pays où les importations sont les plus faibles. Le Mali a presque atteint l'autosuffisance alimentaire. Selon les estimations de la FAO (2017), la consommation moyenne par personne et par an est de 103,2 kg de riz en Sierra Léone, 97,12 en Guinée, $94,75 \mathrm{~kg}$ au Libéria, $72,29 \mathrm{~kg}$ au Sénégal, 63,56 $\mathrm{kg}$ en côte d'ivoire et $56,99 \mathrm{~kg}$ au Mali (Données FAO 2017). La consommation moyenne par tête d'habitant est élevée en riz dans cette espace ciblée avec la forte dominance de la Sierra Leone malgré sa quatrième position en termes de production avec 1,2 millions de tonnes. Par ailleurs, ce niveau élevé de consommation du riz en Afrique de l'Ouest justifie les efforts pour l'accroissement des disponibilités de l'offre locale en riz. Dans certains pays (Côte d'Ivoire, Sénégal, Libéria), le déséquilibre entre l'offre et la demande devient de plus en plus grand et ceci nécessite le recours aux importations. Par contre, ce déséquilibre a tendance à se réduire au Mali et à moindre échelle en Guinée grâce aux productions notées ces dernières années qui ne sont pas cependant fluctuantes selon les saisons. Cette situation conduit aux États à promouvoir la culture du riz dont l'intensification et l'accroissement des productions 

défis en Côte d'Ivoire, Guinée, Libéria, Mali, Sierra Léone et Sénégal

devraient contribuer à améliorer la couverture des besoins.

Marge des acteurs de la chaîne : La marge agrégée des divers acteurs de la chaine de valeur du riz en Afrique est évaluée dans les pays ciblés de la production du paddy à la vente en détail du riz blanc au marché en passant par tous les processus de transformation et de distribution. Ainsi, elle couvre à chaque stade de la chaine les coûts de production, de collecte, d'étuvage, de transformation et de distribution. Les résultats de l'étude en 2016/2017 montrent que la marge est variable selon le pays et la performance des acteurs. Pour la Guinée, le coût de production du riz paddy est de $1311 \mathrm{GNF} /$ ou 0,23 US dollar induisant une marge nette de 1119 GNF ou 0,19 Us dollar par $/ \mathrm{kg}$ de paddy commercialisé du producteur. Le coût moyen de l'étuvage est de 1722 GNF ou 0,29 US dollar / kg et celui de la distribution de 1 $200 \mathrm{GNF} / \mathrm{kg}$. Avec un coefficient moyen de transformation de $57 \%$, le kilo de riz blanc est l'équivalent de $1,754 \mathrm{~kg}$ de paddy. Ainsi avec le prix au détail moyen de 7846 GNF/kg du riz étuvé sur le marché, le coût de production absorbe les $17 \%$. Les commerçants gagnent $4 \%$ du prix en détail, tandis que les transformateurs prennent $10 \%$ et les producteurs $15 \%$. Cette répartition de marge entre acteurs montre l'existence de coûts de production, d'étuvage et d'usinage très élevés. Ceci est induit par les coûts et frais élevés d'achat du paddy, de manutention et des charges de fonctionnement. Ainsi, les marges des divers acteurs restent à des proportions relativement faibles. Néanmoins, il existe de réelles marges de progrès à explorer avec une utilisation efficiente des facteurs de production, d'étuvage, de transformation et de distribution.

En Sierra Leone, le coût de production est en moyenne à 873 Le soit 0,20 US dollar par kg de paddy et la marge nette du producteur estimée à 327 Le ou 0,07 US dollar $/ \mathrm{kg}$. Ainsi le coût de production en Sierra Leone est moins élevé que celui en Guinée, mais la marge nette par $\mathrm{kg}$ reste plus faible de la sous-région. Le coût moyen d'étuvage existant est de $766 \mathrm{Le} / \mathrm{kg}$, tandis que sa marge et de $756 \mathrm{Le} / \mathrm{kg}$ paddy étuvé. Ensuite, le riz est usiné avec un coût de revient pour la décortiqueuse à 136 Le/kg dégageant une marge de 24 Le. Le coût de distribution du riz blanc est de $414 \mathrm{Le} / \mathrm{kg}$ et une marge nette de $66 \mathrm{Le} / \mathrm{kg}$. L'analyse nous indique que tous les opérateurs ont des coûts de production plus élevés que leurs marges. Cela indique une certaine inefficacité dans les opérations.

L'analyse de la chaine de valeur riz au Mali en 2016/2017 révèle un coût de production moyen élevé de 109 FCFA/kg avec un niveau de rendement moyen de 2,7 t/ha, mais avec des pics de 6 t/ha en système irrigué. La performance au niveau de la production est fortement dépendante du niveau des rendements et de la réduction des charges en engrais et produits phytosanitaires ; et la redevance de l'eau en système de maîtrise de l'eau. Dans les bas-fonds et le pluvial, l'accent doit être mis sur l'utilisation des technologies et de la consommation des engrais en vue d'améliorer la productivité rizicole. La marge nette du producteur est ainsi de 45 FCFA/kg représentant $38 \%$ du prix du riz blanc local au marché. Le commerçant gagne à hauteur de $35 \%$ du prix du marché, tandis que les transformateurs sont à $15 \%$. Le commerçant est avantagé par la préférence du consommateur au riz local par rapport au riz importé.

Au Sénégal, le coût de production est évalué à 102 FCFA/kg et le producteur gagne une marge nette de 28 FCFA/kg sur le paddy, soit en moyenne 213423 FCFA/ha avec un itinéraire intensif de consommation d'intrants et un coût hydraulique élevé. Le coût de distribution du paddy est estimé à $4,26 \mathrm{~F} / \mathrm{kg}$, tandis que le coût moyen d'usinage est à 4,55 FCFA/kg de paddy. Dans la répartition du prix du riz blanc en détail au marché entre les divers postes, le coût de production s'octroie la plus grande part soit $58 \%$. II est suivi par la marge nette du producteur qui représente $16 \%$ du prix du riz blanc local. Cela indique un faible profit et une pression pour améliorer son efficience en relation avec une utilisation efficiente des facteurs de production. Les commerçants en gagnent $14 \%$ du prix en détail. Les transformateurs prennent la plus petite portion (soit $6 \%$ ). Cette marge nette apparemment faible des transformateurs s'explique à la fois par la dure compétition dans ce secteur pour l'obtention du paddy et des coûts d'investissements élevés pour la transformation dont l'énergie et par une production en deçà de leur capacité.

Échanges sous-régionaux et potentialités : Comme constaté dans les niveaux annuels de production, l'offre locale du riz dans les pays enquêtés ne couvre qu'entre 20 et $60 \%$ de la demande intérieure. Le Mali constitue cependant, une exception avec une couverture de à $90 \%$ de ses besoins. N'empêche, il importe en moyenne 200000 t/an. Avec une population en Afrique de l'Ouest qui augmente à raison de $3 \%$ en moyenne la demande devient de plus en plus insatisfaite par l'offre locale. II est également qu'une partie du riz local est étuvé (notamment en Guinée, Sierra Leone et Liberia), tandis que la majorité du riz importé est de type non étuvé en provenance des pays Asiatiques. Une analyse de cette situation a permis d'identifier des réseaux d'échanges sous-régionaux du riz. Les experts et personnes 
ressources rencontrées ont fortement confirmé les importations du riz étuvé et non étuvé vers les pays limitrophes des pays étudiés sans données quantitatives (carte 1).

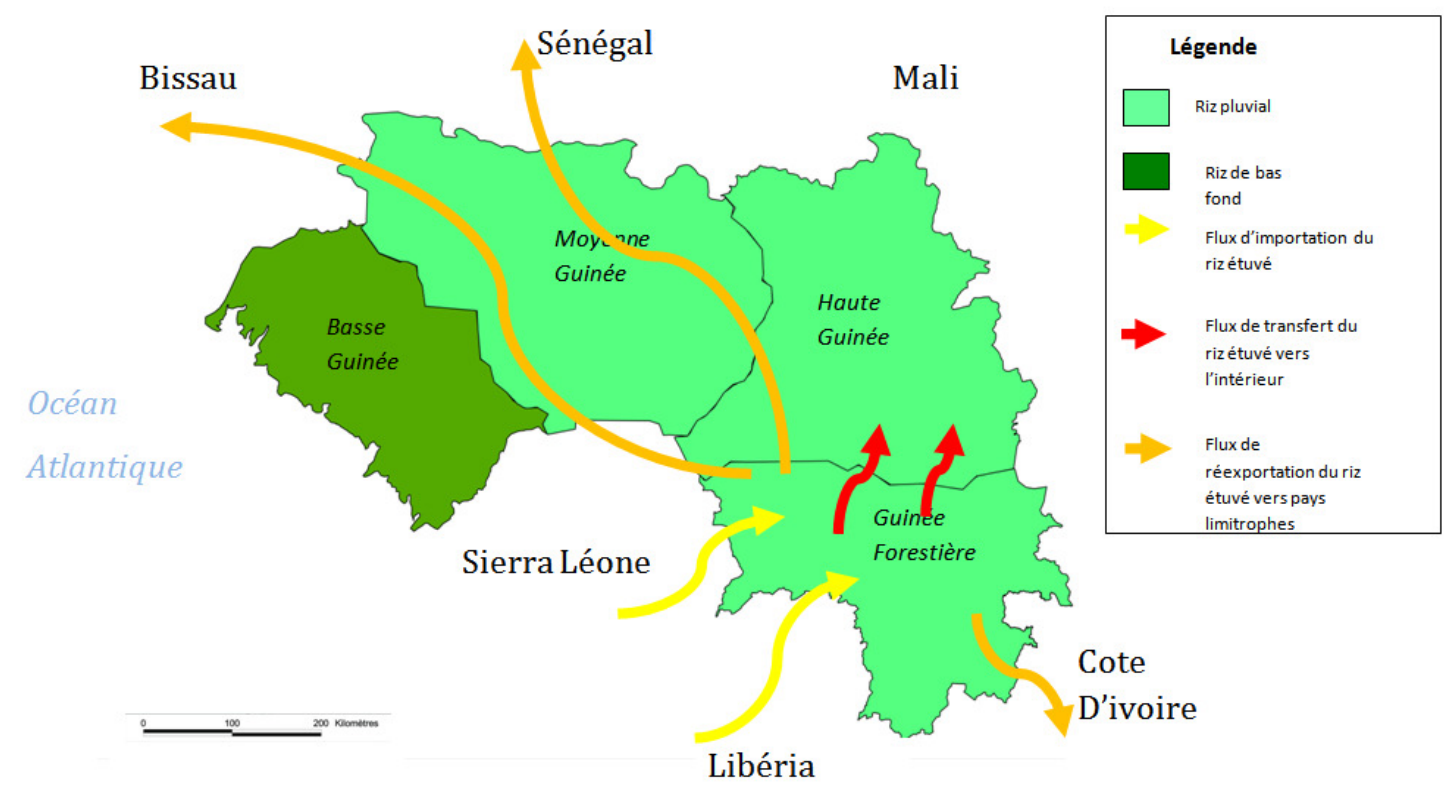

Carte $n^{\circ} 1$ : Cartographie des flux régionaux de commerce du riz

Source : Auteur.

Un regard sur la situation géographique et monétaire des pays étudiés montre que les possibilités de commerce avec en grande partie la monnaie CFA très forte (monnaie relativement plus forte que le franc Guinéen (GNF), le franc Ouguiya de la Mauritanie, la monnaie Leone de la Sierra Leone et le dollar Libérien) sont potentiellement élevées. La moitié des pays ciblés (Cote d'Ivoire, Mali et Sénégal) est ainsi frontalière à ces pays grands consommateurs du riz étuvé (Guinée, Libéria, Burkina, Sierra Léone) et non étuvé (Mauritanie, Gambie). Les traces ainsi identifiées indiquent un potentiel de commerce intrarégional du riz entre ces pays. Cette prédisposition met en évidence les potentiels réseaux de commerce sous-régional. La récente étude CORAF (2014) a montré une offre théorique de 916000 tonnes autour de la Guinée, Sierra Leone, Libéria et Côte d'Ivoire. L'USAID/E-ATP (2010) a également montré un commerce du riz étuvé entre le Burkina, la Côte d'Ivoire, le sud du Sénégal, la Guinée et le Mali avec un potentiel de $100000 \mathrm{t}$ par an. Les données d'exportation du riz de ces six pays évoluent en dents de scie et concernent principalement la Cote d'Ivoire, le Sénégal et le Mali. Entre 2013 et 2016, ces exportations sont en moyenne de 29000 t pour la Cote d'Ilvoire, 5662 t pour le Sénégal et 6 $000 \mathrm{t}$ pour le Mali (FAOSTAT). Des initiatives de commercialisation des céréales dont le riz, ont été mises en œuvre par l'Association Malienne pour la Sécurité et la Souveraineté Alimentaire (AMASSA). Elle a organisé des bourses qui ont favorisé la réalisation de transactions cumulées portant sur au moins sur 300.000 tonnes de céréales dont $30 \%$ en riz entre 2007 à 2016 (AMASSA, 2016). Des bourses internationales ont été créées par I'ONG Afrique Verte (Bamako, 2011; Niamey, 2012 et Ouaga, 2013) avec la participation d'au moins de 9 pays de la sous-région qui a abouti la réalisation en moyenne de 100.000 tonnes de céréales dont environ $20 \%$ en riz (idem, 2016). De façon générale, les bourses permettent de confronter l'offre et la demande et permet d'éliminer plusieurs intermédiaires commerciaux. Les prix issus des bourses sont intéressants pour toutes les parties (offreurs et demandeurs). Les bourses permettent également de garantir la qualité et de fidéliser les relations d'affaires (AMASSA, 2014). Au niveau sous régional, avec l'objectif de favoriser les échanges entre ses états membres, I'UEMOA envisage la mise en place d'une bourse régionale de produits vivriers. Le dispositif envisagé pour la bourse régionale est la création d'une bourse centrale au niveau d'un pays de I'UEMOA et des antennes nationales ou bourses nationales dans chacun des autres pays. Des échanges ou initiatives d'échanges équilibrés sont ainsi notées dans la sous-région. D'une part, ce sont les flux frontaliers du riz local étuvé et de l'autre les flux 

défis en Côte d'Ivoire, Guinée, Libéria, Mali, Sierra Léone et Sénégal

du riz importé et réexporté vers d'autres pays voisins. La nature des rapports économiques entre les pays et les préférences des consommateurs de ces pays, favorisent un commerce apparemment rentable et bénéfique à toutes les parties. Cependant, le volume des exportations de riz blanchi notées en Afrique de l'Ouest reste encore faible, mais indique de potentialités réelles pour développer le commerce intrarégional.

Forces et opportunités de la chaîne de valeur riz en Afrique de l'Ouest : La filière rizicole locale dispose de nombreux atouts. Selon Africarice (2012), le riz est une plante hautement adaptable qui peut être cultivé dans différents écosystèmes rencontrés en Afrique de l'Ouest. Au plan biophysique, l'Afrique de l'Ouest regorge de nombreux avantages en termes de climat, de disponibilité de grandes surfaces de terres exploitables pour la production (disponibilité en quantité et en qualité). En zone pluviale, le potentiel de superficies exploitables est énorme, de même que les marges de progrès. Sur le plan de la recherche, un réseau de centre de recherches travaille à mettre en place des variétés de qualité et d'autres technologies correspondant aux besoins des producteurs et des consommateurs en collaboration avec le Centre du riz pour l'Afrique (AfricaRice). Ceci a abouti à l'introduction de variétés résistantes à la sécheresse, de variétés de riz irrigué à fort potentiel de rendement notamment les variétés SAHEL. Aussi, des variétés de riz de plateaux plus productives (NERICA) ont été introduites au Guinée, Cote d'Ivoire, Sierra Leone et Sénégal et d'autres technologies productives (bonnes pratiques culturales, gestion des sols et des eaux, outils d'aide à la décision.). A cela s'y ajoute une demande soutenue, l'intérêt actuel de divers bailleurs de fonds et, surtout, une prise de conscience par les producteurs que le riz est en passe de devenir la denrée la plus prometteuse du marché (Boutsen et Aertsen, 2013). Des opportunités existent donc pour améliorer les rendements dont la moyenne est estimée à 4 tonnes/ha à l'irriguée qui est largement en dessous du potentiel du matériel végétal disponible qui est de 12 à 13 tha (Ndiaye, 2011). En effet, la zone dispose d'énormes potentialités (eau, terre, vaste marché en mutation) pouvant permettre la création d'une valeur ajouté importante, tant sur le plan de la production que de la transformation ou de la commercialisation. Face à un marché très volatile, capricieux et à une consommation de plus en plus forte, il est nécessaire d'améliorer les systèmes de production. La crise alimentaire de 2008 a été également une opportunité à saisir de la part de tous les acteurs de la chaîne de valeur pour définitivement concurrencer le riz importé. De nouveaux rapports entre l'État et le secteur privé sont apparus, à la faveur de l'augmentation des investissements publics et commerciaux vers l'agriculture en général, et la riziculture en particulier. Dans certains pays, il apparait l'émergence d'une filière locale plus professionnelle. Le secteur privé intervient avec des capacités financières élevées et un engagement d'incitations commerciales. Depuis cette crise, l'Afrique est devenue une destination pour les investissements directs étrangers dans le secteur agricole. De grandes entreprises acquièrent de vastes étendues de terres pour la production de denrées alimentaires et d'autres cultures émergentes. II est évident que les pays africains doivent aborder cette situation avec prudence en raison de la complexité, de la sensibilité politique et des spécifiéscontextuelles de la question foncière à l'intérieur des pays, mais d'un pays à l'autre'. Mais, si ces évolutions sont porteuses d'espoir, elles n'auront pas pour le moment infléchi la dépendance de la région au riz importé, ni la vulnérabilité du marché régional aux évolutions internationales. Le soutien de la recherche et du développement se justifie ainsi que l'implication des structures de financement.

Faiblesses et menaces de la chaîne de valeur riz en Afrique de l'Ouest : Malgré les forces de cette chaîne de valeur, plusieurs faiblesses y sont inhérentes. Le secteur rizicole de la région souffre des inefficiences liées à une productivité faible, et à la cherté de la transformation et de la commercialisation. Selon Lançon (2011), la concurrence entre riz local et riz importé en Afrique de l'Ouest n'est pas seulement biaisée par le jeu des aides directes ou indirectes dont bénéficient les filières d'exportations de riz d'origine asiatique. Elle est aussi faussée par les contraintes qui pèsent sur les modalités de transformation et de commercialisation du riz local : des techniques artisanales, sinon sommaires, pour l'étuvage et le décorticage, des moyens de conditionnement insuffisant et des capacités de financement limitées pour assurer une régularité des flux de riz local auprès des consommateurs urbains. La capacité des filières locales à fournir un riz dont les attributs de marchés sont comparables à ceux des riz importés est un enjeu tout aussi important que le niveau des prix relatifs et la productivité des riziculteurs africains pour améliorer la compétitivité des riz ouest-africains. Dans les pays ciblés, les principaux stress abiotiques et biotiques rencontrés dans les champs des agriculteurs sont les adventices, les oiseaux, les sols (baisse de la

\footnotetext{
${ }^{1}$ Africa Rice (2012). Redynamisation du secteur rizicole en Afrique: une stratégie de recherche pour le développement 2011-2020.
} 

défis en Côte d'Ivoire, Guinée, Libéria, Mali, Sierra Léone et Sénégal

fertilité qui menace les rendements), les inondations, les sols pauvres, la sécheresse, les maladies et les insectes ${ }^{2}$ qui ensemble provoquent une perte moyenne de $22 \%$. L'enquête a également révélé que la riziculture de plateau est affectée par des sécheresses récurrentes, la pauvreté des sols (carences en azote et en phosphore) et l'acidité des sols. Les contraintes socioéconomiques sont, entre autres, l'insuffisance de capitaux, la pression démographique, le faible niveau d'accès au marché, la pénurie de main-d'œuvre et la mauvaise gestion des terres. Cependant, la culture itinérante sur brûlis et le raccourcissement des périodes de jachère ont entraîné une dégradation spectaculaire de la fertilité des sols et une pression accrue des adventices, poussant les agriculteurs à s'étendre vers des zones à plus faible rendement. Selon USAID (2009), malgré l'engagement des gouvernements dans le soutien, le maintien et l'extension des périmètres irrigués publics, il n'y a pas de plan coordonné entre acteurs pour faire correspondre l'emplacement des nouveaux périmètres avec les capacités de développement. L'implication des gouvernements dans la certification des semences et les subventions d'engrais à travers des quasi-monopoles sur l'importation et la distribution sont également considérés comme les principaux obstacles à la modernisation de la chaîne de valeur. Les producteurs de riz d'Afrique de l'Ouest ont des besoins énormes en matière d'investissements de production nécessitant des crédits sur le moyen et le long terme. La majorité des petits producteurs n'ont toutefois pas accès à ces crédits. Le financement du secteur rizicole reste entier malgré les efforts fournis par les États durant ces cinq dernières années. Les banques estiment que le secteur, notamment la production et la transformation sont trop risquées. L'accès à des semences et des fertilisants de qualité et en quantité suffisante est aussi un problème. Le principal point d'achoppement lors de la transformation réside en ce que les producteurs ne parviennent pas à appliquer les normes de qualité élevée qui doivent être respectées afin de pouvoir concurrencer le riz blanc thaillandais ou vietnamien plus pur et non-cassé. Des installations vieillissantes, des entreprises qui ne fonctionnent que trois mois par an, l'absence de stocks permanents et un marketing défaillant (en matière d'emballage, d'étiquettes, de publicité, ...) et la

\footnotetext{
${ }^{2}$ Plus de $90 \%$ des agriculteurs interrogés ont mentionnés ces contraintes.
}

distribution sont autant de défis auxquels les acteurs de la chaine de valeur riz sont confrontés.

Leçons tirées sur la situation de la chaine : En Afrique de l'Ouest, la production de riz a connu une augmentation de $3,23 \%$ par an entre 2013 et 2017 , tandis que le taux de croissance annuel de la population est $3 \%$ (FAO, 2017). Cependant, l'accroissement annuel de la consommation de riz de $4,52 \%$ durant cette période explique en partie la croissance relative de la demande de riz qui reste plus rapide que partout ailleurs dans le monde. Ainsi, l'Afrique de l'Ouest dépend des importations de riz pour faire face à cette situation alimentaire relativement difficile due à une incapacité de la production locale à couvrir les besoins croissants des populations. De façon globale, les importations de riz représentent actuellement environ 5,8 millions de tonnes contre 1,8 millions de tonnes au début des années 1990 (idem, 2017). Cette tendance est assez générale dans tous les pays de la sous-région, même si le poids de certains pays, comme la Côte d'Ivoire, le Nigéria et le Sénégal a fortement influencé cette tendance. L'évolution de cet indicateur indique de façon concluante que la dépendance alimentaire par rapport à cette céréale, céréale synonyme de sécurité alimentaire, n'a pas connu une amélioration malgré les efforts fournis. Cette tendance persiste et la région risque de s'exposer à terme à de nouvelles menaces de crise alimentaire. II est donc évident que des efforts s'imposent pour réduire ce gap qui entraîne une ponction croissante en devises et affecte les perspectives de diversification. Les pays ouest africain doivent donc identifier les zones qui offrent un minimum d'obstacles techniques et les variétés à haut rendement pour enclencher un processus de transformation structurelle de toute la chaîne de valeur riz. La situation de l'offre du riz en Afrique de l'Ouest montre cependant des marges de progrès importantes. En effet, malgré l'accroissement des productions, l'offre ne couvre pas encore la demande. Ensuite, cette offre est tirée par une expansion des superficies et non une amélioration des rendements. Cela indique que le niveau de performance au niveau de l'offre reste insuffisant et ouvre des perspectives avec seulement $50 \%$ du potentiel des terres cultivables qui sont emblavées. Pour le nivellement des rendements au potentiel des variétés améliorées, un besoin de renforcement de capacités techniques et d'utilisation efficiente des technologies se fait sentir. L'amélioration des infrastructures de production est également cruciale, de même que l'utilisation des intrants de qualité dont notamment les semences certifiées et les engrais. Le riz local est en pleine expansion avec un intéressement des consommateurs 

défis en Côte d'Ivoire, Guinée, Libéria, Mali, Sierra Léone et Sénégal

malgré le prix élevé de sa cession. Cela met en évidence que le riz local gagne des parts de marché importantes. Néanmoins, une hausse des importations est observée d'année en année. Ainsi, la demande de riz augmente en Afrique de l'Ouest où les niveaux de consommation per capita s'accroissent et sont entre 57 et $130 \mathrm{~kg}$ par tête et par an. Ces niveaux se situent parmi les plus élevés du monde. Ainsi pour satisfaire cette demande croissante et

\section{CONCLUSION ET RECOMMENDATIONS}

La filière riz dont la matière première (production) offre à la fois, une garantie de nourriture et de numéraire à tous les acteurs du processus à caractère fortement intégrateur. Le riziculteur a des gains, mais les prestataires de service peuvent également bien vivre de la riziculture. La riziculture en Afrique de l'Ouest occupe une position centrale par ses efforts induits sur les activités d'amont et d'aval de la filière et génère des volumes de transactions dix fois supérieurs à son produit brut intrinsèque. Par ailleurs, les évidences empiriques les plus récentes montrent que la chaîne de valeur riz en Afrique de l'Ouest est compétitive. Cependant, la principale question est souvent pourquoi la région continue de dépendre en grande partie du riz importé ? Pourquoi la capacité locale à approvisionner le marché ne progresse pas aussi rapidement que la demande ? Cela s'explique en effet, par de diverses difficultés notamment des frais de transaction élevés et d'une consommation en croissance permanente. Ce diagnostic révèle également que les maillons faibles de la chaîne sont la transformation et la commercialisation. Malgré les efforts

\section{REMERCIEMENT}

Ce projet de recherche a été cofinancé par la Commission de l'Union Économique et Monétaire Ouest Africaine (UEMOA) en partenariat avec AfricaRice « Appui technique aux programmes de mise en œuvre des grandes orientations de la politique agricole de I'Union en vue de la relance du secteur rizicole en Afrique de l'Ouest » et le projet CORAF. Nous tenons ainsi à remercier ces partenaires. En plus, l'auteur remercie Messieurs Pape Makhoudia Mbengue, Selle Cissé Diatta et Talla Tall doctorants à l'Université Gaston Berger de

\section{BIBLIOGRAPHIE}

AfricaRice (2012). Redynamisation du secteur rizicole en Afrique: une stratégie de recherche pour le développement 2011-2020.

ARM (Agence de régulation des Marchés). 2014. Bulletin trimestriel du marché agropastoral Novembre, en riz étuvé préféré, les pays importent du riz des pays asiatiques et du riz étuvé dans la sous-région. Les données statistiques n'existent pas, mais les experts l'évaluent entre 30000 et 100000 tonnes par an. Les consommateurs aisés en zone urbaine acceptent de payer plus cher pour disposer du riz étuvé. Ceci ouvre des perspectives de marché pour le riz local.

fournis, les résultats obtenus au niveau des riziculteurs restent encore faibles. II serait donc important de mettre en place des stratégies d'amélioration en agissant à ces niveaux pour améliorer l'offre du riz local et les valeurs ajoutées créées. II se pose ainsi un besoin d'utilisation efficiente des technologies disponibles. Ceci devrait être accompagné par un accès à la technologie, aux infrastructures productives, au financement et aux cadres de concertation permettant aux acteurs le dialogue et le partage des savoirs. Toutefois, la souveraineté alimentaire des pays ouest africains passera par la maitrise de leurs productions rizicoles et, partant, l'inversion de la balance commerciale déficitaire en raison des importations subséquentes en riz. D'où l'impérieuse nécessité de valoriser les réflexions de mise en commun de plan d'actions concerté pour améliorer la productivité et la compétitivité de la filière rizicole locale. Ce sont là sans doute des conditions pour renforcer l'attractivité du riz local qui, du reste, doit répondre aux attentes des consommateurs et être capable de se mouvoir dans un marché fluctuant.

Saint-Louis, Sénégal pour leur appui à la collecte des informations. L'auteur remercie également l'éditeur de la revue qui a fortement contribué à l'article grâce à ses commentaires précieux. Nous remercions également $\mathrm{Dr}$ Emile Victor Coly, Directeur DPV, Sénégal, Dr Lamissa Diakité, Directeur ECOFIL, IER Mali, Dr Mbene Dieye Faye, PAFA Sénégal, Sékou Diawara, INERA Guinée pour leur concours à la collecte de données et commentaires précieux.

Rapport Système d'Information sur les Marchés, Novembre, $11 p+$ annexes

Boutsen, S. et Aertsen, J. (2013). Peut-on nourrir l'Afrique de l'Ouest avec du riz ? MO Papers, n47-février -- www.mo.be/papers. 
CEDEAO, UEMOA, NEPAD (2014) : Accélérer la mise en œuvre de l'ECOWAP/PDDAA, Programme d'Appui à la mise en œuvre de l'Offensive Régionale pour la relance durable et soutenue de la riziculture en Afrique de l'Ouest ; 96 pages CORAF/WECARD (2014). Analyse de la chaine de valeur du riz en Afrique l'Ouest : pays du Fleuve Mano et le Mali, Rapport Consultation CORAF/WECARD (Fall, A.A et Diakité, L.). Mars, 63p.

Del Villar, P.M. et Bauer, J.M., (2013). Le riz en Afrique de l'Ouest : dynamiques, politiques et perspectives. Cah Agric 22: 336-44. Doi: 10.1684/a9r.2013.0657

FAO. (2017). Small and Medium Agro-Enterprises (SMAEs) and inclusive rice value chains in Africa : Kenya, Tanzania and Senegal, Ongoing research project, (Kelly, S. et al.,) in memo, March, 86p.

Fall, A.A. (2007). Le riz, une denrée stratégique à problème, dossier sur la sécurité ou souveraineté alimentaire, Agrovision, bulletin d'informations trimestriel- CMA/AOC, Mai 2007 $n^{\circ} 3$, PRIECA $/ A O$

Lançon, F. (2011). La compétitivité du riz ouest africain face aux importations : vrais enjeux et fausses questions. Grain de sel, № 54-56.

Lançon, F. (2009): Politique rizicole et sécurisation alimentaire en Afrique de l'Ouest. 3èmes journées en sciences sociales, INRA, SFER et CIRAD, 9, 10 et 11 Décembre 2009-Montpelier France.

Ministère de l'Agriculture et Equipement Rural- DAPSA, Résultats de campagne de Novembre, 19972016.

Ndiaye, S. (2011). La sécurité alimentaire au Sénégal, constats et perspectives ? Contribution GEEN INFO -Revue trimestrielle d'informations environnementales- Décembre 2011, 2p.

USAID (2009). La chaîne de valeur : options stratégiques pour la relance de la filière semencière du Sénégal. USAID/ Projet Croissance économique.

USAID/E-ATP (2010). Chaine de valeur riz étuvé au niveau régional : Étude du marché du riz étuvé entre Burkina Faso, Mali et Sénégal, Rapport Consultation E-ATP (Fall, A.A.), Décembre, 33p.

USDA (2018). Regional Rice Imports, Production, Consumption, and Stocks, Foreign Agricultural Service, Office of Global Analysis, April. 